\title{
Alipoma of the sigmoid colon: a rare cause for intestinal obstruction
}

\author{
Perera MAS, Gunesene DDT \\ Provincial General Hospital, Rathnapura, Sri Lanka
}

Correspondence: Dr.M.A.S.Perera; (akalankashaminda@gmail.com)

\section{Introduction}

Colonic lipomas are non-epithelial, benign, fatty tumors and are uncommon. The reported incidence of colonic lipomas ranges from $0.2 \%$ to $4.4 \%$ (1). Lipomas of the colon were first reported by Bauer in 1757 and are most often located in the ascending colon near the caecum (2). The symptomatic patients with lipomas present with abdominal pain, haemorrhage, diarrhoea, or constipation (3). Lipomas that grow more than $4 \mathrm{~cm}$ in size can lead to obstruction and intussusception. More commonly, definitive diagnosis is made by histopathological staining after the removal of lipoma (4). Surgical resection is recommended to alleviate symptoms and to rule out malignancy (4). In this case report, we describe an intussuscepted, sigmoid colonic lipoma that caused intermittent bowel obstruction.

\section{Case Report}

A 65-years-old previously healthy woman presented with intermittent per rectal fresh bleeding and mucoid discharge for 5 months duration and sub acute intestinal obstruction for 2 days. Her abdominal examination revealed a mass in the left iliac fossa. Her ultrasound scan and CT scan of abdomen revealed an intraluminal lipoma with intussusception within the sigmoid colon (Figure 1).

She underwent sigmoid colectomy, descending colonic and rectal anastomosis \& defunctioning ileostomy followed by subsequent reversal of ileostomy. Gross examination of the specimen revealed a large polypoidal homogeneously yellowish mass, and histological features were consistent with submucosal lipoma in the sigmoid colon (Figure 2).

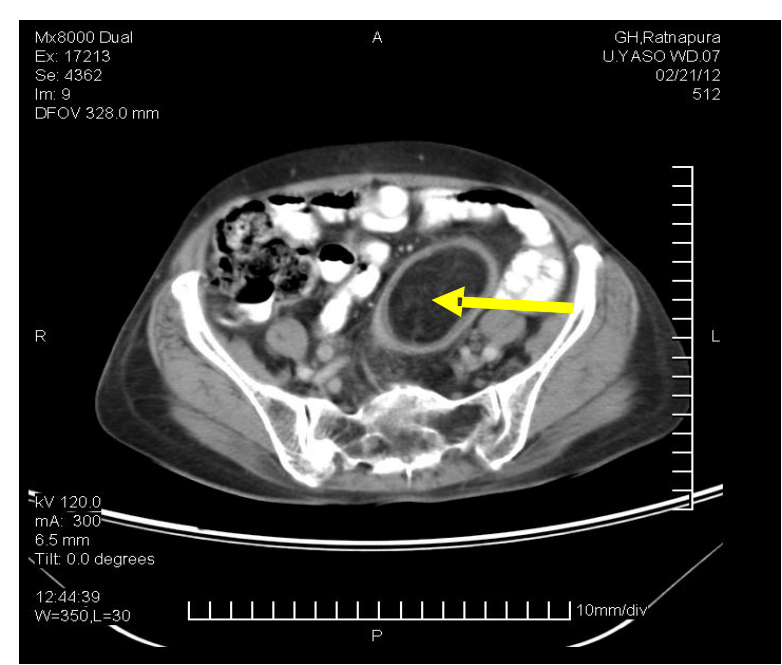

Figure 1: A computed tomography scan of the abdomen showing sigmoid colonic lipoma (yellow arrow)Figure 1: A computed tomography scan of the abdomen showing sigmoid colonic lipoma (yellow arrow)

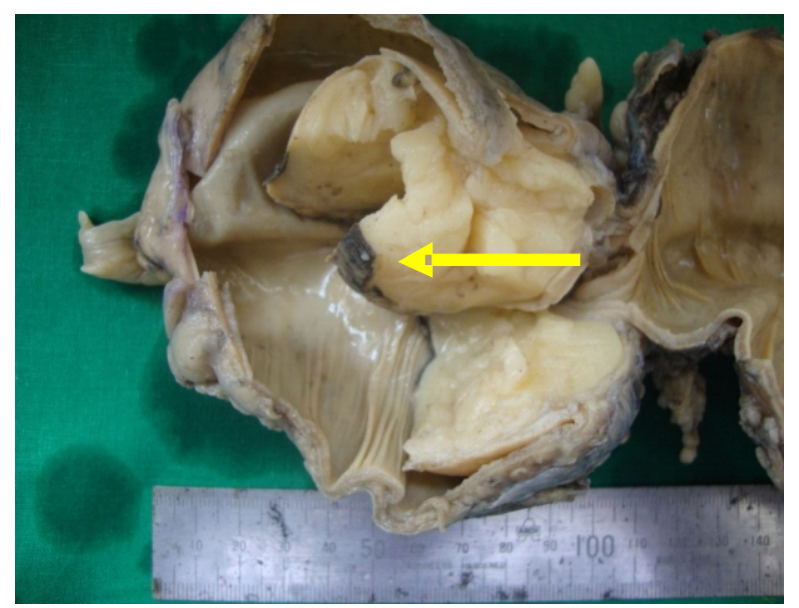

Figure 2: Gross surgical pathology at the resection revealed large polypoidal mass (yellow arrow) 


\section{Discussion}

Large intestinal lipomas are more prevalent in women than in men and have a peak incidence between 50 and 60 years of age. Lipomas of the large intestine are most commonly seen (in order of decreasing frequency) in the caecum, ascending colon, sigmoid colon, descending colon and transverse colon.

The diagnosis can be established radiographically, endoscopically, or surgically. Barium studies are non-diagnostic unless changes in the lipoma's shape and size are seen via a diagnostic test (the squeeze sign) (2). Ninety percent of colonic lipomas are localized to the submucosa; colonic lipomas are rarely found in other layers of the bowel wall (3). Three endoscopic signs can aid in the diagnosis of a lipoma: the "cushion" sign, which occurs when forceps press into the mass, resulting in a depression; the "tenting" sign, which occurs when mucosa is grabbed over the lesion and pulled away, resulting in a tent-like appearance; and the "naked fat" sign, which occurs when fat is grossly extruded after biopsy (5).

The presence of intussusception, intestinal obstruction or suspicion of malignancy warrants surgical resection (4).

As our patient presented with intestinal obstruction, exploratory laparotomy was planned. Unfortunately, the possibility of colonic malignancy could not be precluded and sigmoid colectomy was performed.

\section{Conclusion}

Lipomas of digestive tract are rare and often asymptomatic. But surgical resection is recommended in symptomatic patients or in whom malignancy cannot be ruled out reasonably.

\section{References}

1. Vecchio R, Ferrara M, Mosca F, Ignoto A, Latteri F. Lipomas of the large bowel. European Journal of Surgery 1996; 162(11): 915-9.

2. Mason R, Bristol JB, Petersen V, Lyburn ID. Education and imaging. Gastrointestinal: lipoma induced intussusception of the transverse colon. Journal of Gastroenterology and Hepatology 2010; 25(6): 1177.

3. Ghidirim G, Mishin I, Gutsu E et al. Giant submucosal lipoma of the cecum: report of a case and review of literature. Romanian Journal of Gastroenterology 2005; 14(4): 393-6.

4. Bardaji M, Roset F, Camps R, Sant F, Fernandez-Layos MJ. Symptomatic colonic lipoma: differential diagnosis of large bowel tumors. International Journal of Colorectal Disease 1998; 13(1): 1-2.

5. De Beer RA, Shinya H. Colonic lipomas. An endoscopic analysis. Gastrointestinal Endoscopy 1975; 22(2): 90-1. 